\title{
Aportes de la Comunidad Andina (CAN) en la relación medio ambiente y municipios*
}

\author{
Edwin Francisco Antolinez Sánchez
}

Recibido: 20 de marzo de 2020 • Aprobado: 10 de mayo de 2020

\section{Resumen}

Las autoridades (alcaldes y concejales) de cada municipio deben propender en sus planes de desarrollo por la adecuada protección y conservación del medio ambiente, teniendo en cuenta que es precisamente lo que busca la Comunidad Andina (CAN), Organización de la cual Colombia hace parte, es por ello que el presente artículo pretende resaltar los aportes de la can en la relación medio ambiente y municipios. En este sentido, el problema de investigación que se pretende resolver es determinar si la CAN ha contribuido a mejorar la relación jurídica entre el medio ambiente y los municipios. Todo lo anterior en el marco de una metodología de carácter cualitativo y desde el escenario del método dogmático.

Palabras clave: Comunidad Andina, municipios, derecho comunitario.

\footnotetext{
El presente manuscrito es producto del proyecto de investigación: "Las decisiones y resoluciones emitidas por la Comunidad Andina (CAN) en el marco de protección de derechos del medio ambiente en los municipios", gestionado en el marco del programa de Derecho Administrativo de la Universidad Libre, Sede Bogotá, Colombia.

Citar como: Antolinez Sánchez, E. F. (2020). Aportes de la Comunidad Andina (can) en la relación medio ambiente y municipios. Revista IUSTA, 53, 189-206. Dor: https://doi. org/10.15332/25005286.6275.

** Administrador público egresado de la Escuela Superior de Administración Pública, Territorial Boyacá Casanare (cetap). Tunja. Abogado egresado de la Universidad Santo Tomás, Seccional Tunja, y candidato a magíster en Derecho Administrativo de la Universidad Libre, Sede Bogotá, Colombia. Correo electrónico: edwinfranciscoantolinez@gmail.com. orcid: 0000-0002-3710-8010.
} 


\title{
Contributions of the Andean Community (can) to the Relationship betWeen the EnVironment and Municipalities
}

\begin{abstract}
The authorities (mayors and councilors) of each municipality must promote in their development plans the adequate protection and conservation of the environment, taking into account that this is precisely what the Andean Community (CAN), an organization of which Colombia is a member. This is why this article seeks to highlight the contributions of the CAN to the relationship between the environment and municipalities. In this sense, the research problem to be solved is whether the CAN has contributed to improving the legal relationship between the environment and municipalities. All of the above within the framework of a qualitative methodology and from the scenario of the dogmatic method.
\end{abstract}

Keywords: Andean Community, municipalities, community law.

\section{Contribuições da Comunidade Andina (can) para A RELAÇÃO ENTRE MEIO AMBIENTE E MUNICÍPIOS}

\section{Resumo}

As autoridades (prefeitos e vereadores) de cada município devem orientar seus planos de desenvolvimento à adequada proteção e conservação do meio ambiente, considerando que é justamente o que almeja a Comunidade Andina (CAN), organização da qual a Colômbia faz parte. Por isso, este artigo pretende ressaltar as contribuições da CAN para a relação entre meio ambiente e municípios. Nesse sentido, a pesquisa busca determinar se a CAN vem contribuindo para melhorar a relação jurídica entre o meio ambiente e os municípios. Para isso, foi utilizada metodologia de caráter qualitativo, sob o método dogmático.

Palavras-chave: Comunidade Andina, municípios, direito comunitário. 


\section{Introducción}

A partir de 1972 las preocupaciones ambientales, como resultado de la Conferencia de Estocolmo, comienzan a adquirir mayor trascendencia.

Institucionalmente, el resultado de la Conferencia fue la creación de un Consejo de Administración de los programas relativos al medio ambiente. La Asamblea General de las Naciones Unidas identifica cuatro requerimientos o mecanismos institucionales para el manejo del medio ambiente general: un consejo de gobierno de 58 Estados elegidos por la asamblea, un secretariado, un fondo ambiental, una junta de coordinación ambiental (Carmona, 2000).

Sin duda alguna, el reconocimiento de la comunidad internacional al concepto de medio ambiente es un avance significativo (Cortes, 2013), porque desde su normatividad jurídica entran a tomar fuerza definiciones como ecología, ecosistema, páramos, agua, calentamiento global, entre otros; quedando en evidencia que al contar con un respaldo a nivel mundial va a ser mucho más fácil ampliar la protección y conservación del medio ambiente, pues mientras, por un lado, los medios de comunicación generan publicidad sobre la materia, por otro, resultan interesantes las diversas iniciativas que pueda tener cada país al abordar con mayor profundidad este tema tan importante (Forero, 2014).

A través de las presentes líneas se pretende dar respuesta al siguiente problema de investigación: ¿los aportes de la Comunidad Andina (CAN) contribuyen a un mejoramiento en la relación medio ambiente y municipios?

La Comunidad Andina (en adelante, cAN) es una organización internacional que nace del Acuerdo de Cartagena de 1969 (su tratado constitutivo), es de carácter subregional y está constituida por Estados que en uso de su voluntad decidieron integrarse, amparados en sus intereses colectivos de cooperación e integración económica, social y política (Cobo, 2008).

La temática es pertinente si se tiene en cuenta que con ella se pretende analizar el impacto jurídico de las decisiones de la can en el marco de la relación del derecho colectivo al medio ambiente y los municipios, de tal forma que se abran sendas para futuros estudios, que permitan ahondar en aspectos significativos que trasciendan no solo en el campo del derecho, sino en las demás ramas del conocimiento (Quiroz, 2014).

Se trata de una investigación de tipo cualitativo, porque lo que se pretende es describir, comprender y profundizar sobre un tema en particular, que en este caso 
hace referencia a los aportes de la Comunidad Andina en la relación medio ambiente y municipios.

El alcance del artículo propuesto es descriptivo, porque se analizará y estudiará detalladamente la esencia de las decisiones y resoluciones emitidas por la cAN en lo que se refiere al cuidado del medio ambiente desde los municipios, con el propósito de conocer su impacto a nivel regional en cada uno de los Estados miembros de dicha organización internacional.

Se hará un análisis documental a partir de la información consultada en diferentes libros, tesis y artículos actualizados, tanto físicos como digitales, de los cuales se extraerán los datos más importantes para luego hacer un compendio sobre los mismos, y proceder a generar un nuevo documento de investigación.

\section{Principales aportes de la can desde el escenario de la relación jurídica entre el medio ambiente y los municipios}

En las Constituciones se han consagrado artículos sobre el medio ambiente y el desarrollo sostenible, y en ellas, o en otras normas ambientales, se identifican artículos que hacen referencia a principios que han ido adquiriendo una aceptación universal; ese es el caso, por ejemplo, del derecho de los ciudadanos a disfrutar de un medio ambiente sano y la obligación que tiene el Estado de protegerlo (Blanco, 2017).

En Colombia las actuaciones de las Cortes han servido para mantener intacta la institucionalidad ambiental, ante intentos de la rama ejecutiva para debilitarla como respuesta a las presiones de los entes regulados (Rodríguez, 2001).

Partiendo de lo enunciado en el párrafo anterior, a título personal se considera importante tener en cuenta que en los últimos años han ido apareciendo algunas innovaciones en el derecho ambiental de la CAN, puesto que no solo desde las constituciones políticas, sino también de las leyes de cada país miembro, se propende por el disfrute de un medio ambiente sano y sostenible para las futuras generaciones, produciéndose de esta forma resultados positivos que permiten visualizar un progreso en la normatividad jurídica de la Organización en general, en temas como el calentamiento global, el cuidado de los páramos, la conservación de las fuentes hídricas (océanos, mares, ríos, quebradas y lagos), la protección de los bosques a través de la reducción de talas y quemas indiscriminadas, el uso adecuado de los suelos y la defensa de la fauna. 
La normatividad ambiental andina en mayor evolución es un aporte de la CAN que ayuda a la gestión medio ambiental de los municipios porque permite el progreso de las decisiones y resoluciones en aras de proteger el medio ambiente (Blanco, 2015a).

Lo anterior significa que, a mayores avances normativos, mejores resultados en la tarea de conservar los océanos, los mares, los lagos, los ríos, las quebradas y demás fuentes hídricas que abarcan los municipios pertenecientes a la cAN.

En el marco de la cAN se ha planteado la manera de poder alcanzar un crecimiento económico que mejore la calidad de vida de la población respetando el medio ambiente, incluyendo así las variables que componen el concepto de desarrollo sostenible (Patarroyo y Benavides, 2014).

Los avances en la integración del tema medioambiental en los esfuerzos comunitarios por la promoción del desarrollo sustentable se reflejan en: 1) creación en junio de 1998 del Comité Andino de Autoridades Ambientales (cAaAm), Decisión 435 ; 2) participación en la primera reunión extraordinaria del Foro de Ministros de Medio Ambiente de América Latina y el Caribe, denominada Iniciativa Latinoamericana y Caribeña para el Desarrollo Sostenible; 3) aprobación de los lineamientos para la gestión ambiental y el desarrollo sostenible en la CAN, en 2001, por parte del caAAm (Rodríguez, 2012).

La Decisión 435 de 1998 es de carácter muy relevante, porque no solo crea el Comité Andino de Autoridades Ambientales (CAAAm), sino que establece su conformación por parte de las autoridades nacionales responsables del medio ambiente de cada país miembro, donde los Estados parte designarán un representante titular y uno alterno, quienes serán acreditados por el Organismo Nacional de Integración, a fin de que puedan actuar de mejor en busca del bienestar general (Blanco, 2015b).

La armonización de las políticas ambientales y de desarrollo sostenible es un aporte de la CAN que ayuda a la gestión medio ambiental de los municipios porque los cuatro Estados miembros aplican las mismas directrices. Esto significa que cuando los países que integran determinada organización emplean de manera coherente sus elementos estatales y gubernamentales, el desarrollo del medio ambiente va a tener gran impacto a nivel de sus entidades territoriales (municipios).

Ahora bien, la mayor contribución de la can se evidencia en las emisiones globales: el cambio climático es una paradoja para los países miembros de la CAN (Bolivia, Colombia, Ecuador y Perú). Pese a que sus emisiones de GEI son mínimas en relación con el total mundial, los países de la región comparten un alto riesgo 
de sufrir intensivamente los impactos del cambio climático por la vulnerabilidad de su población y de sus ecosistemas.

El reto de los países andinos en el marco del cambio climático es aparejar el crecimiento económico con el desarrollo social, dando un uso sostenible a sus recursos naturales y minimizando los impactos en el ambiente (cAN, 2007).

Del texto citado, se concluye que el cambio climático es innegable y evidente no solo en los países de la CAN sino en el resto del mundo; su principal causa es la continua emisión de cantidades de petróleo, gasolina y carbón, la tala y quema indiscriminada de bosques y algunos procedimientos de explotación agrícola, actividades resultantes de la inconciencia del ser humano, que no logra imaginar los efectos dañinos a futuro.

Desde el contexto de la biodiversidad se ha establecido una red de coordinación e intercambio de información entre institutos nacionales de cada país miembro de la can. También se han planteado alternativas de desarrollo sustentable, a través del fomento del patrimonio cultural y natural, avanzando en la protección de conocimientos tradicionales, caminos ancestrales andinos y proyectos de ecosistemas compartidos como la conservación de áreas naturales en la frontera ColombiaEcuador-Perú, en departamentos como Nariño y Putumayo que cuentan con abundantes especies animales y vegetales, lo que hace que estas dos entidades territoriales se constituyan como zonas estratégicas en beneficio no solo de Colombia, sino de toda la Comunidad Andina (Blanco, 2014).

Nos habla de ello la Decisión 523 de 1998 que instruye al caAAm que informe cada año al Consejo Andino de Ministros de Relaciones Exteriores, a través de la Secretaría General de la can, sobre los distintos avances obtenidos en la actualización de la Estrategia Regional de Biodiversidad y el desarrollo de los proyectos de desarrollo sostenible y sustentable existentes al respecto.

La biodiversidad es un aporte de la can que ayuda a la gestión medio ambiental de los municipios porque busca la protección y conservación de una gran variedad de especies vegetales y animales que viven en un espacio determinado. Esto significa que cuando las corporaciones autónomas regionales cumplen a cabalidad sus funciones, entre ellas la preservación de la flora y fauna, cada municipio va a contar con más apoyo jurídico para que prevalezca el cuidado del medio ambiente.

Desde el escenario de la prevención y atención de desastres se han desarrollado diversas estrategias, encaminadas a fortalecer la gestión, el establecimiento de 
programas comunes, el intercambio de experiencias e información y la creación de redes y cooperación mutua para proteger los ecosistemas (Blanco, 2013a).

Todo lo anterior ha estado dirigido por el Sistema Nacional para la Prevención y Atención de Desastres (sNPAD) de cada Estado que conforma la can, sistema que abarca todo un conjunto de entidades públicas, privadas y comunitarias, que tienen como objetivo plantear posibles soluciones a los problemas de seguridad de la población que se presenten en su entorno físico por la eventual ocurrencia de fenómenos naturales o antrópicos.

La prevención y atención de desastres es un aporte de la cAN que ayuda a la gestión medio ambiental de los municipios, porque antes de que suceda un fenómeno natural, como por ejemplo un deslizamiento, se adoptan las medidas para mitigarlo.

Lo antes expuesto significa que los alcaldes y concejales deben hacer uso correcto del principio de planeación en aras de evitar sucesos lamentables para la naturaleza, ya que, si no se pueden detener, por lo menos es posible solucionarlos en un tiempo corto y oportuno (Blanco, 2013, b).

En relación con la importancia del agua como líquido de valor, se han establecido estrategias de gestión integrada para la protección y recuperación de los recursos hídricos (CAN, 2010). Lo antes dicho, se sustenta en que los países que integran la CAN cuentan con una riqueza hídrica privilegiada, condiciones geográficas, topográficas y climáticas típicas importantes que determinan un alto promedio anual en las precipitaciones y conforman redes hidrográficas de gran extensión y diversas cuencas que se corresponden con distintos sistemas de uso (Blanco, 2013c).

El agua se encuentra ligada desde tiempos remotos a la constitución de sus culturas, convirtiéndose en el eje fundamental de sus formas de desarrollo y distribución del espacio. La escasez del agua en el mundo se ha convertido en una de las mayores amenazas de la humanidad y la causa de múltiples tensiones y conflictos. Las disputas regionales por las fuentes de agua se incrementan. Al ser esenciales para la supervivencia y el desarrollo, a veces, las reservas de agua dulce han sido el origen de controversias, aunque también son motivo de cooperación entre quienes comparten los recursos hídricos (Ercilio Moura, Rodríguez Chávez y Cabel Noblecilla, 2005).

A la fecha, se ha podido evidenciar un desarrollo equilibrado y armónico de los países miembros de la CAN en materia ambiental. Este organismo pretende contribuir al desarrollo equilibrado y armónico de los países miembros en materia 
ambiental, evitando desigualdades de desarrollo entre cada país miembro y propiciando una distribución equitativa de los beneficios derivados de la integración, de forma coherente, realista y transparente. Se reconoce la existencia de distintas etapas en la aplicación de las políticas que en esta materia propone la CAN.

Los países miembros son conscientes de que se deben disminuir gradualmente las diferencias existentes en la subregión y que hay que apuntarle a un pleno desarrollo equilibrado y armónico a través de la generación de planes, programas y proyectos ambientales que realmente los beneficie a todos, sin embargo, se otorga un tratamiento especial y diferenciado a Bolivia y Ecuador (Aguirre Ochoa, Peña Morales y Massiel Duarte, 2014).

Se valora el esfuerzo realizado por la can en su firme propósito de promover el desarrollo equilibrado y armónico de los países miembros a través de la integración y la cooperación política, económica y social en asuntos ambientales, siendo esto favorable porque, además de cumplir con la agenda, se genera empleo y se fortalece la solidaridad subregional, procurando un mejoramiento en la calidad de vida de los ciudadanos.

El desarrollo equilibrado y armónico de los países miembros de la can en materia ambiental es un aporte de la organización que ayuda a la gestión medioambiental de los municipios, porque cuando existen lazos de amistad entre los pueblos resulta más fácil su progreso integral.

Lo antes expuesto significa que es muy importante que los cuatro países integrantes de la CAN mantengan siempre buenas relaciones económicas, políticas y sociales en aras de que sus municipios, a través del trabajo y las buenas costumbres, cosechen los mejores frutos y propendan por un bienestar general.

La doctrina ha considerado como derechos de la Madre Tierra a los siguientes:

1. Derecho a la vida: es el mantenimiento de la integridad de los sistemas de vida y los procesos naturales que los sustentan, así como las capacidades y condiciones para su regeneración. 2. Derecho al agua: es la preservación de la funcionalidad de los ciclos del agua, de su existencia en la cantidad y calidad necesarias para el sostenimiento de los sistemas de vida, y su protección frente a la contaminación, para la reproducción de la vida de la Madre Tierra y todos sus componentes. 3. Derecho al aire limpio: es la preservación de la calidad y composición del aire para el sostenimiento de los sistemas de vida y su protección frente a la contaminación, para la reproducción de la vida de la Madre Tierra y todos sus componentes. 4. Derecho a vivir libre de contaminación: es la preservación de la Madre Tierra de 
contaminación de cualquiera de sus componentes, así como de residuos tóxicos y radioactivos generados por las actividades humanas. (Contreras, 2012, p. 16)

Dentro de las principales obligaciones de la can, en cada uno de sus niveles y ámbitos territoriales, está el desarrollo de diversas políticas públicas y actuaciones sistemáticas de prevención, protección y precaución para garantizar la prevalencia de los derechos de la Madre Tierra, evitando a toda costa que las acciones humanas conduzcan a la extinción de los seres vivos, la variación de los ciclos y procedimientos que garantizan la vida como derecho fundamental en cada uno de los municipios para el caso de Colombia, o la pérdida de sistemas de vida que incluyen aspectos culturales que hacen parte de la Madre Tierra, el desarrollo de métodos de producción y patrones de consumo para la satisfacción de las necesidades de los pueblos, buscando que vivan bien y resguardando las capacidades institucionales de la cAN.

La Protección de los Derechos de la Madre Tierra es un aporte de la can que ayuda a la gestión medioambiental de los municipios, porque el planeta día a día viene siendo objeto de continuos atentados por parte del ser humano, por ejemplo, cuando se talan y queman indiscriminadamente los bosques nativos.

Lo anteriormente expuesto significa que, si no protegemos desde los municipios a la Madre Tierra, en unos años nuestros herederos se estarán lamentando de la inconciencia del ser humano, cuando el calentamiento global se encuentre en su máximo esplendor y el agua escasee en los páramos y demás ecosistemas.

Es importante resaltar que a la fecha se ha evidenciado un proceso de globalización en la agenda ambiental de la cAN: el proceso de globalización en la agenda ambiental de dicho organismo involucra un desarrollo de las telecomunicaciones a nivel mundial, con el propósito de recortar cada vez más las brechas geográficas que separan a los países y regiones (municipios). En este sentido, la televisión abierta y por cable, inclusive la radio y en general el desarrollo del comercio internacional contribuyen para la difusión y conocimiento de las nomas y políticas de carácter ambiental en las regiones de cada Estado miembro (Villacreces, 2008).

El uso adecuado de las tecnologías de la información y las comunicaciones es un punto clave en la divulgación de las decisiones y resoluciones emitidas por la CAN en lo que corresponde a temas de medio ambiente, toda vez que, al contar con diferentes mecanismos publicitarios se hace más fácil la interacción a nivel regional, ya que las autoridades pueden ingresar rápidamente a la página oficial de la CAN 
y conocer de primera mano cada pronunciamiento que esta profiera, no solo para efectos informativos, sino para ponerlos en práctica de manera inmediata.

Manifiesta lo escrito la Decisión 615 de 2005, la cual crea el Sistema de Información de Notificación y Reglamentación Técnica de la CAN (SIRT) como un instrumento que facilite el cumplimiento de las obligaciones de notificación de reglamentos técnicos y procedimientos de evaluación de la conformidad de los países miembros, y las que se adopten a nivel comunitario. Así como brindar información y atención de consultas que se deriven de dichas notificaciones.

De otro lado, la cAN ha propendido por lograr una economía solidaria tendiente a mejorar los aspectos ambientales. Desde sus inicios, la can buscó la expedición de diversas normas jurídicas con el fin de regular de mejor forma algunas conductas en pro de los aspectos ambientales regionales; así mismo, optó por la liberalización del comercio interregional, la definición de un Arancel Externo Común (AEC), la regulación de la Inversión Extranjera Directa (IED) en la región, la organización de la producción entre los países miembros para impulsar el desarrollo de nuevas industrias y la armonización de las políticas (Creamer 2004).

En este sentido, se resalta la expedición de normas jurídicas por parte de la CAN para cumplir con diferentes finalidades, sin embargo, se considera que, más allá de emitir una cantidad innumerable de reglas que regulen ciertos comportamientos de manera específica, lo que se necesita es hacer cumplir a cabalidad las ya existentes, de tal forma que el ordenamiento jurídico no se torne en la realidad como algo que solamente está escrito, sino que en la práctica sea eficaz al momento de su aplicación.

En relación con los recursos naturales, presentan una extraordinaria riqueza común aportada por la Amazonía, zona de inconmensurable biodiversidad paisajística, florística y variada fauna. Considerada una de las mayores reservas de agua del mundo, con mayores reservas forestales, numerosos recursos estratégicos (titanio, oro, manganeso, bauxita, platino, diamantes), gran reserva en materia energética y biogenética vital para el sostenimiento de la vida humana, estos aspectos hacen de la Amazonía un patrimonio universal y una fortaleza única de la subregión.

Administrar y unificar estrategias para la formación del recurso humano en cuanto al buen uso y sostenibilidad del ecosistema es también una manera de cooperar. Así mismo, esta alianza puede ayudar a resolver parte del desempleo regional sobre todo en las áreas fronterizas de los países limítrofes y volver más coherentes 
las políticas encaminadas a cohesionar culturalmente a los distintos grupos étnicos que la habitan incorporando la ética como ejercicio del bien común (Álvarez, 2001).

De lo expresado por la autora, se tiene que una muestra de unidad e integración al interior de la CAN se da a partir de reconocer la posesión que se tiene de este patrimonio común, el cual hasta ahora ha sido manejado por los países industrializados que con su avanzada capacidad científica han explotado de manera irresponsable las riquezas de la Amazonía, sin acompañar este proceso con la consideración adecuada y sin tener en cuenta el aporte a los países y pueblos indígenas, que tradicionalmente han sido los propietarios y herederos de este patrimonio de la humanidad.

En Colombia, la política general de desarrollo ambiental se mantiene aún a cargo del nivel nacional y la responsabilidad del desarrollo territorial se circunscribe a los límites que la ley establece en las entidades territoriales: municipios, distritos y departamentos. Tales competencias le permiten al municipio asumir proyectos de desarrollo territorial a partir de la valorización de identidad, contando, sin embargo, con recursos limitados. En general, las políticas e instrumentos de desarrollo rural en Colombia no se enfocan hacia la valorización de la identidad territorial de los productos y servicios ofrecidos en las zonas rurales (Soto Uribe y Vargas Vargas, 2008).

Los recursos naturales son un aporte de la CAN que ayudan a la gestión medioambiental de los municipios, porque aquellos son elementos de la naturaleza que contribuyen al bienestar de todos los seres vivos.

Lo anterior significa que, desde el punto de vista económico, los recursos naturales son relevantes para el progreso de los municipios por ayudar a su desarrollo de una manera directa y satisfacer las demandas sociales.

En medio de un periodo de crisis comercial, generada por el TLC negociado por Colombia y Perú con la Unión Europea; y de crisis diplomática, derivada de la ruptura de relaciones diplomáticas entre Colombia y Ecuador en 2008, se planteó una agenda estratégica que buscó orientar el proceso de integración andino.

Dicha agenda, acordada en 2010 por los ministerios de Relaciones Exteriores y de Comercio de los países de la can incluye doce metas: 1) participación ciudadana; 2) política exterior común; 3) integración comercial y complementación económica; 4) integración física y desarrollo de fronteras; 5) desarrollo social; 6) medio ambiente; 7) turismo; 8) seguridad; 9) cultura; 10) cooperación; 11) integración energética y recursos naturales y 12) desarrollo institucional; así como los respectivos 
mecanismos para medir sus niveles de cumplimiento en un plazo de cinco (Guzmán Guzmán y Trejos Trejos, 2017).

En el párrafo anterior se observa la sexta meta consignada en la agenda estratégica andina, que versa sobre el medio ambiente y en ese sentido establece un conjunto de proyectos para entrar a profundizar en la integración de las fronteras andinas, poniendo en marcha los planes y programas de desarrollo en las zonas de frontera y la promoción de acciones tanto públicas como privadas que contribuyan a disminuir las desigualdades latentes entre los Estados miembros de la cAN.

Así lo reafirma la Decisión 759 de 2011 por medio de la cual se emite la Estrategia Andina para mejorar la Eficacia de la Cooperación Internacional para el Desarrollo, la cual en su artículo $1 .^{\circ}$ prevé que con el objetivo de fortalecer la gestión y la coordinación de la cooperación internacional para el desarrollo como instrumento complementario para promover la integración de la subregión y el desarrollo equilibrado y armónico de los países miembros, se aprueba la Estrategia Andina para mejorar la Eficacia de la Cooperación Internacional para el Desarrollo.

El sector minero es solo uno entre muchos otros cuyo desarrollo puede generar impactos significativos al ambiente. Al proponer un análisis del daño ambiental en función de este sector específico no se pretende tomar partido en el ya suficientemente polarizado debate entre ambiente y minería, que por su misma polarización no ha ayudado a construir a favor de ninguno de los dos bandos.

Entendiendo que la protección del ambiente también está amparada por una declaratoria de utilidad pública e interés social y bajo el precepto de minería responsable, el desarrollo de las actividades mineras debe en todo caso atender al cumplimiento de estos objetivos ambientales (Rueda, 2016).

De lo expresado por Rueda (2016) resulta importante agregar que la minería puede ser generadora de grandes perjuicios ambientales cuando esta no es manejada en los términos consagrados en la Constitución y la Ley, es decir, al realizarse de manera ilegal, que es lo que ocurre cotidianamente en los municipios más pequeños del país, y que la clase obrera la denomina minería informal, artesanal o tradicional, pues se convierte en un problema para los alcaldes, porque al entrar a regularla legalmente, sus votantes tienden a sentirse afectados por las medidas que puedan tomar los gobernantes locales (Sänger, 2015).

La inconmensurable biodiversidad y la abundante disponibilidad de recursos naturales y energéticos con los que cuenta América Latina, sumados al carácter 
intercultural de la sociedad civil constituyen, sin lugar a dudas, la principal riqueza de esta región (Pardo, 2014).

Es por ello que no debe sorprendernos que, desde la aprobación a inicios de los años setenta del siglo pasado de los primeros instrumentos internacionales en materia medioambiental, los Estados latinoamericanos hayan abrazado rápidamente la "causa ecológica", dando inicio a un paulatino proceso de "institucionalización" de la gestión del medio ambiente y, en consecuencia, a un notorio incremento de la normativa ambiental (tanto sustantiva como adjetiva) en todos los niveles legislativos (llegándose incluso a su “constitucionalización”), que por sus desarrollos reconoce muy pocos parangones en el ámbito del derecho comparado (Gallego, 2014).

Según Esborraz (2016), América Latina cuenta con incalculables riquezas naturales, tales como el petróleo, el oro, el diamante, la esmeralda, entre otros; los cuales se encuentran en países como Colombia, Perú, Bolivia y Ecuador, que conforman la cAN. Sin embargo, se nota cómo la inadecuada exploración y explotación de estos hidrocarburos y minerales ha llevado a catástrofes naturales irreparables que en muchas ocasiones han desencadenado incluso en casos de responsabilidad patrimonial del Estado (Palomares y Calonje, 2015).

Ahora bien, el calentamiento global genera una serie de transformaciones en la calidad ambiental y la oferta de los recursos naturales en los distintos ámbitos territoriales (Barragán, 2016). Es en este punto donde se hace necesario establecer que metodológicamente se apela en este texto a la noción de territorio como el ámbito de correlación dinámica de los distintos actores y sus intereses acerca de la apropiación del mismo (Vega, 2014).

La legislación nacional ubica a los Bosques Nacionales dentro de la flora silvestre, es decir, el conjunto de plantas no cultivadas por el hombre. Además de su condición silvestre, se caracteriza porque sus especímenes son maderables.

Cabe resaltar que una de las maneras más eficaces para proteger los bosques nacionales es la declaración de zonas de protección ambiental, puesto que ellas restringen los usos del suelo e incluso limitan el derecho a la propiedad de los particulares con el objeto de proteger y preservar los recursos naturales allí contenidos. Al respecto, la legislación ha señalado tres tipos de áreas: las Zonas de Reserva Forestal (ZRF), el sistema de parques naturales y el área especial ecológica (Rodríguez, 2015).

Así lo manifiesta la Decisión 391 de 1996 por medio de la cual se emite el Régimen Común sobre Acceso a los Recursos Genéticos, la cual en su 
considerando 1 prevé que "los Países Miembros son soberanos en el uso y aprovechamiento de sus recursos, principio que ha sido ratificado además por el Convenio sobre Diversidad Biológica suscrito en Río de Janeiro en junio de 1992 y refrendado por los cinco Países Miembros".

El concepto páramo es tan complejo que es difícil definirlo. El páramo es un ecosistema, un bioma, un paisaje, un área geográfica, una zona de vida, un espacio de producción e inclusive un estado del clima. Además, el valor y el significado del mismo pedazo de páramo pueden ser muy distintos para el campesino que pasta sus animales o para el biólogo que estudia un bicho dentro de la paja. Esta complejidad de sentidos y de visiones refleja la gran importancia del páramo, pero a la vez es el origen de muchos malentendidos y hasta de malas intenciones por parte de ciertos grupos de interés. De la misma manera, por ser un término tan complejo, descriptivo de diferentes sujetos similares y con anotaciones históricas, académicas, políticas y culturales, es muy difícil definir lo que realmente es un páramo (Medina y Mena 2001).

Los páramos son un aporte de la Comunidad Andina (CAN) que ayuda a la gestión medio ambiental de los municipios, porque se trata de importantes ecosistemas con superficies llanas, poco fértiles y desérticas que suelen estar ubicadas varios metros sobre el nivel del mar.

Lo anterior significa que los páramos en los municipios son fuente de vida, pues conservan grandes cantidades de agua a través de sus musgos y frailejones que además los hacen importantes en un área forestal determinada (Garzón, 2013).

\section{Conclusiones}

Es evidente que los aportes de la cAN contribuyen a un mejoramiento en la relación medio ambiente y municipios, porque los mismos han sido estudiados detalladamente por expertos en la materia, considerando las costumbres y arraigos de cada Estado miembro, de tal forma que las diferencias entre países no se conviertan en una barrera que impida su aplicación.

La CAN es un organismo de carácter internacional que en la actualidad agrupa cuatro países que trabajan coordinadamente en pro de un bienestar común, razón por la cual sus aportes en materia ambiental son muy valiosos, teniendo en cuenta que los mismos persiguen un desarrollo integral y que en un momento determinado pueden ser tenidos en cuenta por otras organizaciones. 
La protección de los derechos de la Madre Tierra, la institucionalización y constitucionalización del medio ambiente, el cuidado de los bosques naturales y la conservación de los páramos; se consideran algunos de los aportes más importantes de la CAN en materia ambiental, sin dejar de lado otras contribuciones relevantes que nacen desde los municipios.

\section{Referencias}

Aguirre, J., Peña, M. y Massiel, S. (2014). La Comunidad Andina: un paradigma de integración económica en Latinoamérica. Managua, Nicaragua. Recuperado el 29 de octubre de 2019 de file://C:/Users/USUARIO/Downloads/Dialnet-LaComunidadAndina-5109429\%20 (9).pdf

Álvarez, R. (2001). Cooperación internacional, integración y capital social. Un marco interpretativo desde la Comunidad Andina. San Cristóbal, Venezuela. Recuperado el 31 de octubre de 2019 de http://www.saber.ula.ve/bitstream/handle/123456789/20862/ articulo4.pdf?sequence=1\&isAllowed $=y$

Barragán, D. (2016). La construcción de la mentalidad democrática como necesidad en el posconflicto. Revista Via Inveniendi et Iudicandi, 11(1), 37-57.

Blanco, C. (2013a). Aproximación a la noción de soberanía estatal en el marco del proceso andino de integración. Revista Republicana, 15, 91-103.

Blanco, C. (2013b). Algunas propuestas frente a la crisis de legitimidad de la Comunidad Andina. Revista Análisis Politico, 26(78), 141-155.

Blanco, C. (2013c). La Comunidad Andina en el marco de lo jurídico y político en Revista Prolegómenos. Derechos y Valores, 26(31), 78-90.

Blanco, C. (2014). La crisis de la Comunidad Andina. Revista de Derecho, 42. 1-32.

Blanco, C. (2015a). La influencia positiva de la cAN en la descentralización territorial colombiana. Revista Opinión Jurídica, 14(27), 161-174.

Blanco, C. (2015b). La conexión entre descentralización territorial colombiana e integración andina. Revista Análisis Politico, 28(84), 78-98.

Blanco, C. (2017). Condiciones de cohesión entre la Comunidad Andina (can) y la descentralización territorial colombiana. Revista Republicana, 23(23), 67-92 Recuperado de http://ojs.urepublicana.edu.co/index.php/revistarepublicana/article/view/406 
Carmona, M. d. (2000). Derechos en relación con el medio ambiente. México, México. Recuperado el 11 de noviembre de 2019 de https://books.google.com.co/books?id=1 OUvueSgZcEC\&printsec=frontcover\&hl=es\&source=gbs_ge_summary_r\&cad=0\#v= onepage\&q\&f=false

Carnero, V. (2004). Comunidad internacional y cambio climático global. Buenos Aires, Argentina. Recuperado el 2 de noviembre de 2019 de https://racimo.usal.edu.ar/5758/1/ P\%C3\%A1ginas\%20desdeTesis.UNI\%270045434.Comunidad\%20Internacional\%20 y\%20cambio\%20clim\%C3\%A1tico\%20global.pdf

Cobo, A. (2008). Las relaciones comerciales de la Comunidad Andina. Barcelona, España. Recuperado el 19 de mayo de 2020 de https://eulacfoundation.org/es/system/files/ Las\%20relaciones\%20comerciales\%20de\%20la\%20Comunidad\%20Andina\%20de\%20 Naciones\%20Estudio\%20espec\%C3\%ADfico\%20de\%201a\%20negociaci\%C3\%B3n\%20 del\%20Acuerdo\%20de\%20Asociaci\%C3\%B3n.pdf

Comunidad Andina (CAN) (2007). Prioridades de la Comunidad Andina ante el cambio climático. Lima, Perú. Recuperado el 16 de octubre de 2019 de http://www.comunidadandina. org/StaticFiles/OtrosTemas/MedioAmbiente/libro_donde_comenzamos.pdf

Comunidad Andina (CAN) (2010). Comunidad Andina: situación actual y principales desafios. Lima, Perú. Recuperado el 16 octubre de 2019 de https://www.cepal.org/sites/default/ files/events/files/presentacion_ana_maria_tenenbaum3.pdf

Contreras, A. (2012). Gestión ambiental en los países de la Comunidadd Andina. Lima, Perú. Recuperado el 30 de octubre de 2019 de http://www.comunidadandina.org/StaticFiles/ OtrosTemas/MedioAmbiente/revista9.pdf

Cortes, S. (2013). La globalización económica y los derechos humanos. Revista Via Inveniendi et Iudicandi, 8(2), 138-149.

Ercilio, F., Rodríguez, S., Cabel, W., Ortiz, I., Noriega, P. y Tejada, M. (2005). Desafíos del derecho humano al agua en el Perú. Lima, Perú. Recuperado el 4 de febrero de 2020 de http://www.ingenieroambiental.com/4030/libroaguaedicion2.pdf

Esborraz, D. (2016). El modelo ecológico alternativo latinoamericano entre protección del derecho bumano al medio ambiente y reconocimiento de los derechos de la naturaleza. Bogotá D.C., Colombia. Recuperado el 11 de noviembre de 2019 de file://C:/Users/USUARIO/ Downloads/4575-Texto\%20del\%20art\%C3\%ADculo-19972-1-10-20160728.pdf

Estrada Oyuela, R. A. y Zeballos de Sisto, M. C. (1993). Evolución reciente del derecho ambiental internacional. Buenos Aires, Argentina: A-Z Editora S. A. 
Forero, J. (2014). El valor superior de la justicia en la Unión Europea. Especial referencia al Estado constitucional social y democrático de derecho español. Revista Verba Iuris, 31, 91-114. Recuperado el 5 de junio de 2018 de https://revistas.unilibre.edu.co/index. php/verbaiuris/article/view/59/53

Gallego, J. (2014). Paradoja y complejidad de los derechos humanos en la sociedad moderna. Sentido y comunicación. Revista IUSTA, 40, 143-165.

Garzón, E. (2013). Globalización del derecho, fetichismo legal el velo de los derechos humanos. Revista Verba Iuris, 30, 56-78.

Guzmán, C. y Trejos, L. (2017). Más allá de las fronteras. Bogotá D. C., Colombia: Universidad del Norte. Recuperado el 3 de noviembre de 2019 de https://www.bokus.com/ bok/9789587417715/mas-alla-de-las-fronteras-integracion-vecindad-y-gobernanza/

Medina, G. y Mena, P. (2001). Los páramos en el Ecuador. Particularidades, problemas y perspectivas. Ecuador Quito. Editorial Abya Yala y Proyecto Páramo.

Palomares, J. y Calonje, N. (2015). Tratados de libre comercio Colombia-Asia: cuestión preliminar y perfiles de negociación. Revista IUSTA, 43(1), 17-41.

Pardo, N. (2014). Un recorrido por los derechos colectivos en la jurisprudencia argentina. Revista Via Inveniendi et Iudicandi, 9(1), 32-49. Recuperado el 2 de enero de 2018 de http://revistas.usantotomas.edu.co/index.php/viei/article/view/1338/1540

Patarroyo, S. y Benavides, P. (2014). Rupturas significantes: revisiones críticas en torno al derecho. Revista Via Inveniendi et Iudicandi, 9(1), 7-31.

Quiroz, M. (2014). Acercamiento a las "oposiciones paradigmáticas” entre neoconstitucionalismo y positivismo jurídico. Revista IUSTA, 41(2), 77-97.

Rodríguez, G. y Páez, I. (2015). Temas de derecho ambiental: una mirada desde lo público. Bogotá D. C., Colombia. Recuperado el 13 de noviembre de 2019 de https://editorial. urosario.edu.co/pageflip/acceso-abierto/temas-de-derecho-ambiental.pdf

Rodríguez, D. (2012). El desarrollo sustentable de la cuenca amazónica en la agenda ambiental de la Comunidad Andina. Quito, Ecuador. Recuperado el 16 de octubre de 2019 de https://media.proquest.com/media/hms/ORIG/1/kgXSC?_s=vQdFWMEdOt94\%2 FoLaquZvcWDwEOM\%3D

Rodríguez, M. (2001). Los avances de la gestión ambiental en los países de la Comunidad Andina, 10 años después de la Conferencia de Río de Janeiro Sobre Medio Ambiente y Desarrollo. Quito, Ecuador. Recuperado el 15 de octubre de 2019 de http://www.manuelrodriguezbecerra. org/bajar/avancesg.pdf 
Rueda, M. (2016). La desatención hacia el daño ambiental en Colombia. Bogotá D. C., Colombia. Recuperado el 11 de noviembre de 2019 de https://editorial.urosario.edu.co/ gpd-la-desatencion-hacia-el-dano-ambiental-en-colombia.html

Sänger, R. (2015). La garantía de la propiedad y el principio de proporcionalidad como límites de la carga tributaria en Alemania. Revista IUSTA, 42(1), 73-99.

Soto, D. y Vargas, J. (2008). Valorización de la identidad territorial, politicas públicas y estrategias de desarrollo territorial en los países de la Comunidad Andina. Bogotá D. C., Colombia. Recuperado el 31 de octubre de 2019 de file://C:/Users/USUARIO/Downloads/ document\%20(1).pdf

Vega, J. (2014). Aproximaciones conceptuales básicas a la adaptación al cambio climático en Colombia. Bogotá D. C., Colombia. Recuperado el 13 de noviembre de 2019 de file://C:/Users/ USUARIO/Downloads/141-Texto\%20del\%20art\%C3\%ADculo-566-1-10-20190404. pdf

Villacreces, F. (2008). La marca notoria en la cAN. Quito, Ecuador. Recuperado el 31 de octubre de 2019 de http://repositorio.uasb.edu.ec/bitstream/10644/1161/1/SM84Villacreses-La\%20marca\%20notoria\%20en\%201a\%20CAN.pdf 